\title{
A Boundary Estimate for Singular Sub-Critical Parabolic Equations
}

\author{
Ugo Gianazza \\ Dipartimento di Matematica "F. Casorati", Università di Pavia \\ via Ferrata 5, 27100 Pavia, Italy \\ email: gianazza@imati.cnr.it \\ Naian Liao \\ Fachbereich Mathematik, Universität Salzburg \\ Hellbrunner Str. 34, 5020 Salzburg, Austria \\ email: naian.liao@sbg.ac.at
}

\begin{abstract}
We prove an estimate on the modulus of continuity at a boundary point of a cylindrical domain for local weak solutions to singular parabolic equations of $p$ laplacian type, with $p$ in the sub-critical range $\left(1, \frac{2 N}{N+1}\right]$. The estimate is given in terms of a Wiener-type integral, defined by a proper elliptic $p$-capacity.
\end{abstract}

Mathematics Subject Classification (2020): Primary 35K67, 35B65; Secondary 35B45, 35K20

Key Words: Parabolic $p$-laplacian, boundary estimates, elliptic p-capacity, continuity, Wiener-type integral

\section{Introduction}

Let $E$ be an open set in $\mathbb{R}^{N}$, and for $T>0$ let $E_{T}$ denote the cylindrical domain $E \times[0, T]$. Moreover let

$$
S_{T}=\partial E \times(0, T], \quad \partial_{p} E_{T}=S_{T} \cup(\bar{E} \times\{0\})
$$

denote the lateral, and the parabolic boundary respectively.

We shall consider quasi-linear, parabolic partial differential equations of the form

$$
u_{t}-\operatorname{div} \mathbf{A}(x, t, u, D u)=0 \quad \text { weakly in } E_{T},
$$

where the function $\mathbf{A}(x, t, u, \xi): E_{T} \times \mathbb{R}^{N+1} \rightarrow \mathbb{R}^{N}$ is assumed to be measurable with respect to $(x, t) \in E_{T}$ for all $(u, \xi) \in \mathbb{R} \times \mathbb{R}^{N}$, and continuous with respect to $(u, \xi)$ for a.e. $(x, t) \in E_{T}$. Moreover, we assume the structure conditions

$$
\left\{\begin{array}{l}
\mathbf{A}(x, t, u, \xi) \cdot \xi \geq C_{o}|\xi|^{p} \\
|\mathbf{A}(x, t, u, \xi)| \leq C_{1}|\xi|^{p-1}
\end{array} \quad \text { a.e. }(x, t) \in E_{T}, \forall u \in \mathbb{R}, \forall \xi \in \mathbb{R}^{N}\right.
$$


where $C_{o}$ and $C_{1}$ are given positive constants, and we take

$$
1<p \leq \frac{2 N}{N+1} \stackrel{\text { def }}{=} p_{*} .
$$

The number $p_{*}$ is referred to as a critical value in the local regularity theory for equations (1.1)-(1.2) (see [6]). The range (1.3) is often called singular, sub-critical, whereas $p_{*}<p<2$ is singular, super-critical.

In addition, the principal part $\mathbf{A}$ is assumed to be monotone in the variable $\xi$ in the sense

$$
\left(\mathbf{A}\left(x, t, u, \xi_{1}\right)-\mathbf{A}\left(x, t, u, \xi_{2}\right)\right) \cdot\left(\xi_{1}-\xi_{2}\right) \geq 0
$$

for all variables in the indicated domains, and Lipschitz continuous in the variable $u$, that is,

$$
\left|\mathbf{A}\left(x, t, u_{1}, \xi\right)-\mathbf{A}\left(x, t, u_{2}, \xi\right)\right| \leq \Lambda\left|u_{1}-u_{2}\right|\left(1+|\xi|^{p-1}\right)
$$

for some given $\Lambda>0$, and for the variables in the indicated domains.

Important examples of partial differential equations satisfying (1.1)-(1.5) include the parabolic $p$-laplacian

$$
u_{t}-\operatorname{div}\left(|D u|^{p-2} D u\right)=0
$$

and more generally

$$
u_{t}-\sum_{i, j=1}^{N}\left(a_{i j}(x, t)|D u|^{p-2} u_{x_{i}}\right)_{x_{j}}=0,
$$

where $\left(a_{i j}\right)$ is a positive definite $N \times N$ matrix with bounded and measurable entries.

Let us consider a boundary datum

$$
\left\{\begin{array}{l}
g \in L^{p}\left(0, T ; W^{1, p}(E)\right) \\
g \text { continuous on } \bar{E}_{T} \text { with modulus of continuity } \omega_{g}(\cdot) .
\end{array}\right.
$$

We are interested in the boundary behavior of solutions to the Cauchy-Dirichlet problem

$$
\left\{\begin{array}{l}
u_{t}-\operatorname{div} \mathbf{A}(x, t, u, D u)=0 \quad \text { weakly in } E_{T} \\
\left.u(\cdot, t)\right|_{\partial E}=g(\cdot, t) \quad \text { a.e. } t \in(0, T] \\
u(\cdot, 0)=g(x, 0),
\end{array}\right.
$$

with $g$ as in (1.6). We do not impose any a priori requirements on the boundary of the domain $E \subset \mathbb{R}^{N}$.

Notice that when $p$ is in the sub-critical range (1.3), the boundedness of a weak solution to (1.7) is not guaranteed by the mere notion of weak solution. A detailed discussion is given, for example in [5, Chapter V], or in [6, Chapter 6, Section 21.3]. Therefore, we directly assume the boundedness of solutions.

We refer to the parameters $\left\{p, N, C_{o}, C_{1}\right\}$ as our structural data, and we write $\gamma=\gamma\left(p, N, C_{o}, C_{1}\right)$ if $\gamma$ can be quantitatively determined a priori only in terms of the above quantities. The generic constant $\gamma$ may change from line to line. 
For $x_{o} \in \mathbb{R}^{N}$ and $\rho>0, K_{\rho}\left(x_{o}\right)$ denotes the cube of edge $2 \rho$, centered at $x_{o}$ with faces parallel to the coordinate planes. When $x_{o}$ is the origin of $\mathbb{R}^{N}$, we simply write $K_{\rho}$.

Let $\left(x_{o}, t_{o}\right) \in S_{T}$, and for $R_{o} \in(0,1)$ we set the backward, space-time cylinder

$$
Q_{R_{o}}=K_{R_{o}}\left(x_{o}\right) \times\left(t_{o}-2 R_{o}^{p}, t_{o}\right],
$$

where $R_{o}$ is so small that $\left(t_{o}-2 R_{o}^{p}, t_{o}\right] \subset(0, T]$. Moreover, we define

$$
\mu_{o}^{+}=\underset{Q_{R_{o}} \cap E_{T}}{\operatorname{ess} \sup } u, \quad \mu_{o}^{-}=\underset{Q_{R_{o}} \cap E_{T}}{\operatorname{essinf}} u, \quad \omega_{o}=\mu_{o}^{+}-\mu_{o}^{-}=\underset{Q_{R_{o}} \cap E_{T}}{\operatorname{ess} \operatorname{Osc}} u .
$$

We will give the formal definition of solution to (1.7) in $\S 2.1$. Now we proceed to state the main result of this paper.

Theorem 1.1. Let $u$ be a bounded, weak solution to (1.7), and assume that (1.2)-(1.6) hold. Then there exist positive constants $\gamma, c, \alpha$, and $q_{o}>\frac{1}{p-1}$, which depend only on the data $\left\{p, N, C_{o}, C_{1}\right\}$, such that

$$
\underset{Q_{\rho}\left(\omega_{o}\right) \cap E_{T}}{\operatorname{ess} \operatorname{osc}} u \leq \omega_{o} \exp \left\{-\gamma \int_{\rho^{\alpha}}^{1}[\delta(s)]^{q_{o}} \frac{d s}{s}\right\}+2 \underset{\widetilde{Q}_{o}(\rho) \cap S_{T}}{\operatorname{osc}} g,
$$

where $0<\rho<R_{o}$,

$$
\begin{gathered}
Q_{\rho}\left(\omega_{o}\right)=K_{\rho}\left(x_{o}\right) \times\left(t_{o}-\frac{1}{2} c \omega_{o}^{2-p} \rho^{p}, t_{o}\right], \\
\delta(\rho)=\frac{\operatorname{cap}_{p}\left(K_{\rho}\left(x_{o}\right) \backslash E, K_{\frac{3}{2} \rho}\left(x_{o}\right)\right)}{\operatorname{cap}_{p}\left(K_{\rho}\left(x_{o}\right), K_{\frac{3}{2} \rho}\left(x_{o}\right)\right)},
\end{gathered}
$$

$\widetilde{Q}_{o}(\rho)$ is a proper reference cylinder, which shrinks to $\left(x_{o}, t_{o}\right)$ as $\rho \rightarrow 0$, and $\operatorname{cap}_{p}(D, B)$ denotes the (elliptic) p-capacity of $D$ with respect to $B$.

Remark 1.1. In $\S 3$ we will give a more precise quantification of $q_{o}$. Its value depends on the Harnack-type inequality we will present in Theorem 2.1.

Remark 1.2. Although the precise expression of $\widetilde{Q}_{o}(\rho)$ plays no role, as far as the decay of $u$ at the boundary is concerned, we present it here for the reader's convenience:

$$
\widetilde{Q}_{o}(\rho) \stackrel{\text { def }}{=}\left\{\begin{array}{l}
K_{2 \tilde{r}}\left(x_{o}\right) \times\left[t_{o}-c \omega_{o}^{2-p} 2(2 \tilde{r})^{p}, t_{o}\right] \\
\text { with } \tilde{r}=\left[\bar{\omega}\left(\rho^{\alpha}\right)\right]^{\frac{1}{2}},
\end{array}\right.
$$

where $(0,1) \ni \rho \mapsto \bar{\omega}(\rho)$ is defined by

$$
\bar{\omega}(\rho)=\exp \left\{-\int_{\rho}^{1}[\delta(s)]^{q_{o}} \frac{d s}{s}\right\} .
$$

We refer to $[9, \S 6.4]$ for the derivation of $\widetilde{Q}_{o}(\rho)$. 
A point $\left(x_{o}, t_{o}\right) \in S_{T}$ is called a Wiener point if $\int_{\tau}^{1}[\delta(\rho)]^{q_{o}} \frac{d \rho}{\rho} \rightarrow \infty$ as $\tau \rightarrow 0$; see Appendix $\mathrm{A}$ for more. Relying on this definition, from Theorem 1.1 we can conclude the following corollary in a standard way.

Corollary 1.1. Let $u$ be a bounded, weak solution to (1.7), assume that (1.2)-(1.6) hold true, and that $\left(x_{o}, t_{o}\right) \in S_{T}$ is a Wiener point. Then

$$
\lim _{\substack{(x, t) \rightarrow\left(x_{o}, t_{o}\right) \\(x, t) \in E_{T}}} u(x, t)=g\left(x_{o}, t_{o}\right) .
$$

Theorem 1.1 also implies Hölder regularity up to the boundary under a fairly weak assumption on the domain, i.e. the complement of $E$ is uniformly p-fat. More precisely, a set $A$ is uniformly $p$-fat, if for some $\gamma_{o}, \bar{\rho}>0$ one has

$$
\frac{\operatorname{cap}_{p}\left(K_{\rho}\left(x_{o}\right) \cap A, K_{\frac{3}{2} \rho}\left(x_{o}\right)\right)}{\operatorname{cap}_{p}\left(K_{\rho}\left(x_{o}\right), K_{\frac{3}{2} \rho}\left(x_{o}\right)\right)} \geq \gamma_{o}
$$

for all $0<\rho<\bar{\rho}$ and all $x_{o} \in A$. See [14] for more on this notion. However, we point out next that this conclusion can be achieved merely under the structural condition (1.2) of $\mathbf{A}$, for all $1<p<2$.

Corollary 1.2. Let $u$ be a bounded, weak solution to (1.7), assume that (1.2) holds true for $1<p<2$, that the complement of the domain $E$ is uniformly $p$-fat, and let $g$ be Hölder continuous. Then the solution $u$ is Hölder continuous up to the lateral boundary.

\subsection{Method of the Proof}

A boundary estimate of the kind (1.8) was established for the elliptic $p$-laplacian by Maz'ya [16. The method employed was a potential theoretical one and heavily depended on the comparison principle. Such an estimate of Maz'ya was later established for very general elliptic operators with structures similar to (1.2) by Gariepy and Ziemer 8]. Our approach mainly follows their ideas whose adaption to the parabolic $p$-laplacian setting presents considerable difficulty, cf. [9, 10]. For this reason, we recapitulate the main steps in the following. For simplicity, let us suppose $u$ is a solution to the elliptic $p$-laplacian in an open set $E \subset \mathbb{R}^{N}$ and it attains zero on part of the boundary $O \subset \partial E$.

The first step consists in showing the truncated function $(u-k)_{+}$with $k>0$ is a local sub-solution in a cube $K_{2 \rho}\left(x_{o}\right)$ with the center $x_{o} \in O$, after a zero extension outside $E$. Then we work with the non-negative, local super-solution $v$ in $K_{2 \rho}\left(x_{o}\right)$ defined by

$$
v \stackrel{\text { def }}{=} \mu-(u-k)_{+}, \quad \text { where } \mu=\sup _{K_{2 \rho}\left(x_{o}\right)}(u-k)_{+} .
$$

Here and in the sequel, we use "sup/inf" instead of "ess sup/ess inf" for simplicity. 
Next, we derive an energy estimate for $v$ of the following type:

$$
\int_{K_{\frac{3}{2} \rho}\left(x_{o}\right)}|D(v \zeta)|^{p} d x \leq \gamma \mu \rho^{N-p}\left(f_{K_{\frac{3}{2} \rho}\left(x_{o}\right)} v d x\right)^{p-1},
$$

where $\zeta \in C_{o}^{1}\left(K_{\frac{3}{2} \rho}\left(x_{o}\right)\right)$ is a cutoff function that equals 1 in $K_{\rho}\left(x_{o}\right)$. Notice that $v \zeta \in W_{o}^{1, p}\left(K_{\frac{3}{2} \rho}\left(x_{o}\right)\right)$ and $v \zeta=\mu$ in $K_{\rho}\left(x_{o}\right) \backslash E$. Hence by the definition of $p$-capacity in Appendix $\mathrm{A}$ we may estimate

$$
\int_{K_{\frac{3}{2} \rho}\left(x_{o}\right)}|D(v \zeta)|^{p} d x \geq \mu^{p} \operatorname{cap}_{p}\left(K_{\rho}\left(x_{o}\right) \backslash E, K_{\frac{3}{2} \rho}\left(x_{o}\right)\right) .
$$

Combining (1.10) and (1.11) and noting $\operatorname{cap}_{p}\left(K_{\rho}\left(x_{o}\right), K_{\frac{3}{2} \rho}\left(x_{o}\right)\right)=c \rho^{N-p}$ for some positive $c=c(p, N)$, we easily obtain

$$
\mu[\delta(\rho)]^{\frac{1}{p-1}} \leq \gamma f_{K_{\rho}\left(x_{o}\right)} v d x .
$$

Finally, the reduction of oscillation is realized via an application of the weak Harnack inequality for the non-negative super-solution $v$ in $K_{2 \rho}\left(x_{o}\right)$. As a result we arrive at

$$
\mu[\delta(\rho)]^{\frac{1}{p-1}} \leq \gamma f_{K_{\rho}\left(x_{o}\right)} v d x \leq \widetilde{\gamma} \inf _{K_{\rho}\left(x_{o}\right)} v
$$

which implies

$$
\sup _{K_{\rho}\left(x_{o}\right)}(u-k)_{+} \leq \mu\left(1-\frac{1}{\gamma}[\delta(\rho)]^{\frac{1}{p-1}}\right) .
$$

Coming back to our parabolic setting, the analog of (1.9) is defined in (2.1), which will be proven to be a super-solution across the lateral boundary in Lemma 2.2. The analog of (1.12) has been proven in [9] and will be recalled in Lemma 2.3. Due to the lack of a proper weak Harnack inequality in the singular, sub-critical range of $p$, we will instead employ a Harnack-type inequality presented in Theorem 2.1] This is the stage where we need the comparison principle.

\subsection{Novelty and Significance}

This is the third paper in a wider project devoted to the study of boundary behavior of solutions to (1.7): in [9] we dealt with the singular super-critical range $p \in\left(p_{*}, 2\right)$, in [10] we studied the degenerate case $p>2$, and here we consider the singular subcritical interval given by $p \in\left(1, p_{*}\right]$, which to our knowledge has never been dealt with before. Some remarks about the significance of our results have already been given in [9, 10, and we will not repeat them here: what we are going to concentrate on, are the differences that the sub-critical range shows with respect to the super-critical one.

In all these three papers, the most interesting result is that a Wiener point is a continuity point for the solution at the boundary, and a quantitative characterization 
of the decay in a neighborhood of that point at the boundary is given (see (1.8) in this case). However, there is a fundamental difference: for $p>p_{*}$ (see [9, 10]), a point $\left(x_{o}, t_{o}\right) \in S_{T}$ is a Wiener point if $\int_{0}^{1}[\delta(\rho)]^{\frac{1}{p-1}} \frac{d \rho}{\rho}=\infty$, whereas here we require $\int_{0}^{1}[\delta(\rho)]^{q_{o}} \frac{d \rho}{\rho}=\infty$, with $q_{o}>\frac{1}{p-1}$. Hence, our result is not optimal, and our quantitative estimates cannot recover what is already known qualitatively, at least in the prototype case.

Indeed, the fact that a Wiener point with $q_{o}=\frac{1}{p-1}$ is a continuity point has already been observed in [3, Proposition 5.4] for the prototype parabolic $p$-laplacian

$$
u_{t}-\operatorname{div}\left(|D u|^{p-2} D u\right)=0,
$$

for any $p>1$, hence both singular, i.e. with $1<p<2$, and degenerate, i.e. with $p>2$ (see also [12]). In [3] the characterization is provided in terms of a family of barriers, adapting to the parabolic $p$-laplacian the well-known Perron method. This approach is quite flexible, as it allows us to give some simple geometric conditions which ensure the regularity of boundary points. Here by regular, we mean a boundary point where all solutions to the Dirichlet problem attain their continuous boundary values continuously. Non-cylindrical domains can then be considered, and indeed Proposition 5.4, mentioned above, deals with quite general sets, of which cylinders as the ones considered here are just a particular instance. On the other hand, Perron's method, at least as developed in [3], does not give any quantitative modulus of continuity. Moreover, at the moment it is not known, whether the qualitative, geometric characterization of regular points is true for more general operators $\mathbf{A}$, like the ones we study here.

The lack of optimality of the exponent $q_{o}$ notwithstanding, here the novelty with respect to the existing literature is twofold: first of all, we deal with quite general operators, and the only restriction for Theorem 1.1 and Corollary 1.1 lies in the requirement that the Comparison Principle is satisfied, whereas for Corollary 1.2 the structural condition (1.2) suffices; on the other hand, as we have already mentioned above, to our knowledge a quantitative characterization, in the singular sub-critical range, of the boundary behavior of solutions to (1.7) for rough sets has never been provided.

There is yet another new observation regarding what we had in [9]: we stated Theorem 1.1 of [9] in centered cylinders. Here we seize this opportunity to point out that in fact backward cylinders will also work in [9], just like in Theorem 1.1 here. The modification can be modeled on Lemma 3.1 below. This matches the observation made in [3, Theorem 3.1] for the prototype equation (1.13), that what happens in the future, namely for $t>t_{o}$, does not affect the regularity of the boundary point $\left(x_{o}, t_{o}\right)$ for the parabolic $p$-laplacian (1.13).

Although we have basically followed the same approach as in [9], the fundamental difference lies in the Harnack inequalities we have to employ. When $p \in\left(p_{*}, 2\right)$, we can rely upon an intrinsic weak Harnack inequality, and this allows us to conclude; when $p \in\left(1, p_{*}\right]$, only Harnack-type estimates are at disposal (see Theorem 2.1 below), and this is ultimately the reason for the lack of optimality of $q_{o}$. It is important to recall 
that the lack of a proper Harnack inequality when $p \in\left(1, p_{*}\right]$, is a structural fact, as suitable counterexamples in [4] and [6, Chapter 6] show. On the other hand, we think that the non-optimality of $q_{o}$ given here is just a technical fact, and a different approach might provide $q_{o}=\frac{1}{p-1}$, in such a way yielding a unified description for solutions to (1.1) -(1.2), in the full singular range $1<p<2$. Unfortunately, we could not come up with such an approach at the moment.

Finally, few words about the contents of the paper. The proof of Theorem 1.1 is given in $\S$ 3 and of Corollary 1.2 in $\S$, in both sections we concentrate on the actual novelties, and we refer to the analogous proof in [9], whenever it would be a straightforward repetition of arguments already displayed elsewhere.

We devote $\S 2$ to introductory materials, in particular an $L^{1}$ Harnack inequality; the remaining sections concern the discussion of an auxiliary problem ( $\S$ 2.4 $)$, the presentation of the Harnack-type inequality which is known in the singular sub-critical range $(\S 2.5)$, and a lower bound for a proper super-solution, proven in [9] (§ 2.6) $)$. An Appendix collects definitions, results, and some examples about the notion of capacity.

Acknowledgement. U. Gianazza is grateful to the TIFR-CAM of Bangalore, India, where part of this work was written. U. Gianazza was supported by the grant 2017TEXA3H_002 "Gradient flows, Optimal Transport and Metric Measure Structures". N. Liao was supported by the FWF-Project P31956-N32 "Doubly nonlinear evolution equations". Both authors are grateful to the anonymous referees for their comments, which greatly helped improve the quality of the paper.

\section{Preliminaries}

\subsection{The Definition of Solutions}

A function

$$
u \in C\left(0, T ; L_{\mathrm{loc}}^{2}(E)\right) \cap L_{\mathrm{loc}}^{p}\left(0, T ; W_{\mathrm{loc}}^{1, p}(E)\right)
$$

is a local, weak sub(super)-solution to (1.1)-(1.2) if for every compact set $K \subset E$ and every sub-interval $\left[t_{1}, t_{2}\right] \subset(0, T]$

$$
\left.\int_{K} u \varphi d x\right|_{t_{1}} ^{t_{2}}+\int_{t_{1}}^{t_{2}} \int_{K}\left[-u \varphi_{t}+\mathbf{A}(x, t, u, D u) \cdot D \varphi\right] d x d t \leq(\geq) 0
$$

for all non-negative test functions

$$
\varphi \in W_{\mathrm{loc}}^{1,2}\left(0, T ; L^{2}(K)\right) \cap L_{\mathrm{loc}}^{p}\left(0, T ; W_{o}^{1, p}(K)\right) .
$$

This guarantees that all the above integrals are convergent. A function $u$, which is both a local, weak sub-solution and a local, weak super-solution, is a local, weak solution.

For any $k \in \mathbb{R}$, let

$$
(v-k)_{-}=\max \{-(v-k), 0\}, \quad(v-k)_{+}=\max \{v-k, 0\} .
$$


Accordingly, we notice that

$$
k-(u-k)_{-}=\min \{u, k\}, \quad k+(u-k)_{+}=\max \{u, k\} .
$$

Using (1.2) 1 and employing a similar method as in $\left(\mathbf{A}_{6}\right)$ of [ [5, Chapter II] or Lemma 1.1 of [6. Chapter 3], we can show the equation (1.1) with (1.2) is parabolic, in the sense that

$$
\left\{\begin{array}{l}
\text { whenever } u \text { is a local weak sub(super)-solution, } \\
\text { the function } k \pm(u-k)_{ \pm} \text {is a local weak sub(super)-solution, for all } k \in \mathbb{R} \text {. }
\end{array}\right.
$$

A weak sub(super)-solution to the Cauchy-Dirichlet problem (1.7) is a measurable function

$$
u \in C\left(0, T ; L^{2}(E)\right) \cap L^{p}\left(0, T ; W^{1, p}(E)\right)
$$

satisfying

$$
\int_{E} u \varphi(x, t) d x+\iint_{E_{T}}\left[-u \varphi_{t}+\mathbf{A}(x, t, u, D u) \cdot D \varphi\right] d x d t \leq(\geq) \int_{E} g \varphi(x, 0) d x
$$

for all non-negative test functions

$$
\varphi \in W^{1,2}\left(0, T ; L^{2}(E)\right) \cap L^{p}\left(0, T ; W_{o}^{1, p}(E)\right) .
$$

In addition, we take the boundary condition $u \leq g(u \geq g)$ to mean that $(u-g)_{+}(\cdot, t) \in$ $W_{o}^{1, p}(E)\left((u-g)_{-}(\cdot, t) \in W_{o}^{1, p}(E)\right)$ for a.e. $t \in(0, T]$. A function $u$, which is both a weak sub-solution and a weak super-solution, is a weak solution.

We have given the definition in a global way, but all the following arguments and results will have a local thrust: indeed, what we are interested in, is whether solutions $u$ to (1.7) continuously assume the given boundary data at a single point or on some distinguished part of the lateral boundary $S_{T}$ of a cylinder, but not necessarily on the whole $S_{T}$. In this context, the initial datum does not play a role.

In the sequel we will need the following comparison principle for weak (sub/super)solutions (see [12, Lemma 3.1], [13, Lemma 3.5] and [3, Section 2]). We let $\Omega$ be an open set in $\mathbb{R}^{N}$, and $\Omega_{S}:=\Omega \times[0, S]$. Note carefully that there is no connection between $\Omega$ and the set $E$.

Lemma 2.1 (Weak Comparison Principle). Suppose that $v$ is a weak super-solution and $u$ is a weak sub-solution to (1.7) in $\Omega_{S}$ under conditions (1.2)-(1.6), and with boundary values $g$ and $h$ respectively. If $v$ and $-u$ are lower semicontinuous on $\overline{\Omega_{S}}$ and $g \geq h$ on the parabolic boundary $\partial_{p} \Omega_{S}$, then $v \geq u$ a.e. in $\Omega_{S}$.

Remark 2.1. As remarked in [12], the proof of the Weak Comparison Principle shows that a super-solution $v$ is greater than a sub-solution $u$ in $\Omega_{S}$ if $u(x, 0) \leq v(x, 0)$ and for each $t \in(0, S)$ the function $x \mapsto(u(x, t)-v(x, t))_{+}$is in the space $W_{o}^{1, p}(\Omega)$. See also [6, Chapter 7]. 


\subsection{Particular Super-solutions Across $S_{T}$}

Fix $\left(x_{o}, t_{o}\right) \in S_{T}$, consider the cylinder

$$
Q=K_{32 \rho}\left(x_{o}\right) \times\left[s, t_{o}\right]
$$

where $s$ and $t_{o}$ are such that $0<s<t_{o} \leq T$, and let $\Sigma \stackrel{\text { def }}{=} S_{T} \cap Q$.

We extend $\mathbf{A}$ to $\widetilde{\mathbf{A}}$ defined in $Q \times \mathbb{R} \times \mathbb{R}^{N}$, setting

$$
\widetilde{\mathbf{A}}(x, t, u, \xi)=\left\{\begin{array}{lll}
\mathbf{A}(x, t, u, \xi) & \text { for a.e. } & (x, t) \in Q \cap E_{T}, \forall u \in \mathbb{R}, \forall \xi \in \mathbb{R}^{N}, \\
|\xi|^{p-2} \xi & \text { for a.e. } & (x, t) \in Q \backslash E_{T}, \forall u \in \mathbb{R}, \forall \xi \in \mathbb{R}^{N} .
\end{array}\right.
$$

It is apparent that $\widetilde{\mathbf{A}}$ satisfies conditions (1.2) and (1.4)-(1.5) in $Q \times \mathbb{R} \times \mathbb{R}^{N}$, with $C_{o}$ and $C_{1}$ replaced by $\min \left\{1, C_{o}\right\}$ and $\max \left\{1, C_{1}\right\}$ respectively.

We have the following simple lemma, which has been stated in [9, Lemma 2.1]. However, we present a complete proof here.

Lemma 2.2. Take any number $k$ such that $k \geq \sup _{\Sigma} g$. Let $u$ be a weak solution to the problem (1.7), and define

$$
u_{k}= \begin{cases}(u-k)_{+}, & \text {in } Q \cap E_{T}, \\ 0, & \text { in } Q \backslash E_{T}\end{cases}
$$

Then $u_{k}$ is a weak sub-solution to (1.1) in the cylinder $Q$ with $\mathbf{A}$ replaced by $\widetilde{\mathbf{A}}(x, t, k+$ $\left.u_{k}, D u_{k}\right)$. The same conclusion holds for the zero extension of $u_{h}=(h-u)_{+}$for truncation levels $h \leq \inf _{\Sigma} g$ with $\mathbf{A}$ replaced by $-\widetilde{\mathbf{A}}\left(x, t, h-u_{h},-D u_{h}\right)$.

Proof. This is a boundary version of the arguments in $\left(\mathbf{A}_{6}\right)$ of [5, Chapter II] or Lemma 1.1 of [6. Chapter 3]. For $\ell>0$, let $\llbracket u \rrbracket_{\ell}$ denote the Steklov average of $u$ in the time variable. In terms of the Steklov averages, the weak formulation of the solution $u$ to (1.7) can be written as

$$
\int_{E \times\{t\}} \partial_{t} \llbracket u \rrbracket_{\ell} \varphi d x+\int_{E \times\{t\}} \llbracket \widetilde{\mathbf{A}}(x, t, u, D u) \rrbracket_{\ell} \cdot D \varphi d x=0
$$

for all $0<t<T-\ell$ and for all $\varphi \in W_{o}^{1, p}(E) \cap L^{\infty}(E)$ (see [5, Chapter II, Remark 1.1] for more details on this equivalent formulation).

Let us omit the reference to $x_{o}$. Consider an arbitrary interval $\left[t_{1}, t_{2}\right] \subset\left[s, t_{o}\right]$. Let $\zeta$ be a piecewise smooth function in $K_{32 \rho} \times\left(t_{1}, t_{2}\right)$, vanishing on $\partial K_{32 \rho} \times\left(t_{1}, t_{2}\right)$. It is rather easy to show that the following is an admissible test function for $\varepsilon>0$ :

$$
\left\{K_{32 \rho} \cap E\right\} \times\left(t_{1}, t_{2}\right) \ni(x, t) \mapsto \varphi(x, t)=\frac{\left(\llbracket u \rrbracket_{\ell}-k\right)_{+}}{\left(\llbracket u \rrbracket_{\ell}-k\right)_{+}+\varepsilon} \zeta .
$$

Moreover, since $\varphi(\cdot, t)$ vanishes on $K_{32 \rho} \cap \partial E$ in the sense of traces for a.e. $t \in\left(t_{1}, t_{2}\right)$ ([9, Lemma 2.1]), we may extend such $\varphi$ to be zero outside $E_{T}$, and carry over the 
integral formulation into $Q$. This remark together with a time integration in $\left(t_{1}, t_{2}\right)$ gives

$$
\int_{t_{1}}^{t_{2}} \int_{K_{32 \rho}} \partial_{t} \llbracket u \rrbracket_{\ell} \varphi d x d t+\int_{t_{1}}^{t_{2}} \int_{K_{32 \rho}} \llbracket \widetilde{\mathbf{A}}(x, t, u, D u) \rrbracket_{\ell} \cdot D \varphi d x d t=0
$$

The first term is estimated via an integration by parts, to obtain

$$
\begin{aligned}
\int_{t_{1}}^{t_{2}} \int_{K_{32 \rho}} \partial_{t} \llbracket u \rrbracket_{\ell} \varphi d x d t=\int_{t_{1}}^{t_{2}} \int_{K_{32 \rho}} \partial_{t} \mathfrak{h}\left(\llbracket u \rrbracket_{\ell}, \varepsilon\right) \zeta d x d t \\
=\left.\int_{K_{32 \rho}} \mathfrak{h}\left(\llbracket u \rrbracket_{\ell}, \varepsilon\right) \zeta d x\right|_{t_{1}} ^{t_{2}}-\int_{t_{1}}^{t_{2}} \int_{K_{32 \rho}} \mathfrak{h}\left(\llbracket u \rrbracket_{\ell}, \varepsilon\right) \partial_{t} \zeta d x d t
\end{aligned}
$$

where we have set

$$
\mathfrak{h}\left(\llbracket u \rrbracket_{\ell}, \varepsilon\right)=\int_{k}^{\llbracket u \rrbracket_{\ell}} \frac{(s-k)_{+}}{(s-k)_{+}+\varepsilon} d s .
$$

The second term is estimated by

$$
\begin{aligned}
\int_{t_{1}}^{t_{2}} & \int_{K_{32 \rho}} \llbracket \widetilde{\mathbf{A}}(x, t, u, D u) \rrbracket_{\ell} \cdot D \varphi d x d t \\
& =\int_{t_{1}}^{t_{2}} \int_{K_{32 \rho}} \llbracket \widetilde{\mathbf{A}}(x, t, u, D u) \rrbracket_{\ell} \cdot\left[D \zeta \frac{\left(\llbracket u \rrbracket_{\ell}-k\right)_{+}}{\left(\llbracket u \rrbracket_{\ell}-k\right)_{+}+\varepsilon}+\zeta \frac{\varepsilon D\left(\llbracket u \rrbracket_{\ell}-k\right)_{+}}{\left(\left(\llbracket u \rrbracket_{\ell}-k\right)_{+}+\varepsilon\right)^{2}}\right] d x d t .
\end{aligned}
$$

Combining all the terms we arrive at

$$
\begin{aligned}
\int_{K_{32 \rho}} \mathfrak{h} & \left.\left(\llbracket u \rrbracket_{\ell}, \varepsilon\right) \zeta d x\right|_{t_{1}} ^{t_{2}}-\int_{t_{1}}^{t_{2}} \int_{K_{32 \rho}} \mathfrak{h}\left(\llbracket u \rrbracket_{\ell}, \varepsilon\right) \partial_{t} \zeta d x d t \\
& +\int_{t_{1}}^{t_{2}} \int_{K_{32 \rho}} \llbracket \widetilde{\mathbf{A}}(x, t, u, D u) \rrbracket_{\ell} \cdot D \zeta \frac{\left(\llbracket u \rrbracket_{\ell}-k\right)_{+}}{\left(\llbracket u \rrbracket_{\ell}-k\right)_{+}+\varepsilon} d x d t \\
& =-\varepsilon \int_{t_{1}}^{t_{2}} \int_{K_{32 \rho}} \zeta \llbracket \widetilde{\mathbf{A}}(x, t, u, D u) \rrbracket_{\ell} \cdot \frac{D\left(\llbracket u \rrbracket_{\ell}-k\right)_{+}}{\left(\left(\llbracket u \rrbracket_{\ell}-k\right)_{+}+\varepsilon\right)^{2}} d x d t .
\end{aligned}
$$

The conclusion is reached by first letting $\ell \rightarrow 0$ and then $\varepsilon \rightarrow 0$. One only has to notice that the right-hand side produces a non-positive quantity in this process, due to the ellipticity of $\widetilde{\mathbf{A}}$.

Let $k$ be any number such that $k \geq \sup _{\Sigma} g$, and for $u_{k}$ as in Lemma 2.2, set

$$
\left\{\begin{array}{l}
\mu=\sup _{Q} u_{k} \\
v: Q \rightarrow \mathbb{R}_{+}, \quad v \stackrel{\text { def }}{=} \mu-u_{k}
\end{array}\right.
$$

It is not hard to verify that $v$ is a non-negative, weak super-solution to (1.1) in $Q$. More precisely, we can write

$$
v_{t}-\operatorname{div}(-\widetilde{\mathbf{A}}(x, t, k+\mu-v,-D v)) \geq 0 \quad \text { weakly in } Q .
$$


Remark 2.2. As shown in the proof, any extension of $\mathbf{A}$ outside $E_{T}$ would work for our purpose, provided it verifies the similar structure conditions (1.2) and (1.4)-(1.5). For simplicity, in the following we will denote by $\mathbf{A}$ the extended function $\widetilde{\mathbf{A}}$.

Remark 2.3. The choice of $k$ in the definition of $u_{k}$ is done in order to guarantee that $u_{k}$ can be extended to zero in $Q \backslash E_{T}$ : this yields a function which is defined on the whole $Q$, and is needed in Lemma 2.3. Therefore, any other choice of $k$ which ensures the same extension of $u$ to the whole $Q$ is allowed.

\subsection{An $L^{1}$ Harnack Inequality for Solutions when $1<p<2$}

As we have already done when stating Lemma 2.1 , let $\Omega$ be an open set in $\mathbb{R}^{N}$, and $\Omega_{S}:=\Omega \times[0, S]$. As before, that there is no connection between $\Omega$ and the set $E$.

Proposition 2.1. Suppose $u$ is a non-negative, local weak solution to (1.1)-(1.2) in $\Omega_{S}$ for $1<p<2$. There exists a positive constant $\gamma$ depending only on the data $\left\{p, N, C_{o}, C_{1}\right\}$, such that for all $\left[s_{1}, t_{1}\right] \subset[0, S]$, and $K_{2 \rho}(y) \subset \Omega$

$$
\sup _{s_{1}<\tau<t_{1}} \int_{K_{\rho}(y)} u(x, \tau) d x \leq \gamma \inf _{s_{1}<\tau<t_{1}} \int_{K_{2 \rho}(y)} u(x, \tau) d x+\gamma\left(\frac{t_{1}-s_{1}}{\rho^{\lambda}}\right)^{\frac{1}{2-p}}
$$

where

$$
\lambda=N(p-2)+p .
$$

The constant $\gamma=\gamma(p) \rightarrow \infty$ either as $p \rightarrow 2$ or as $p \rightarrow 1$.

Proof. See Proposition A.1.1 in [6, Appendix A]. Here conditions (1.4)-(1.5) are not needed in the proof.

\subsection{An Auxiliary Problem}

Assume $\eta$ and $\bar{T}$ satisfy

$$
s<\eta<\eta+\bar{T} \leq t_{o}
$$

with $s, t_{o}$ as in the definition of $Q$ in $\S 2.2$. Suppose $u_{o}$ is a non-negative, bounded, measurable function defined in $K_{32 \rho}\left(x_{o}\right)$, with support in $K_{2 \rho}\left(x_{o}\right)$. We consider the Cauchy-Dirichlet problem

$$
\left\{\begin{array}{l}
u_{\tau}-\operatorname{div} \mathbf{A}(x, \tau+\eta, u, D u)=0 \quad \text { weakly in } K_{32 \rho}\left(x_{o}\right) \times(0, \bar{T}] \\
\left.u(\cdot, \tau)\right|_{\partial K_{32 \rho}\left(x_{o}\right)}=0 \quad \text { a.e. } \tau \in(0, \bar{T}] \\
u(\cdot, 0)=u_{o}
\end{array}\right.
$$

We may apply (2.2) to the above solution in the pair of cubes $K_{2 \rho}\left(x_{o}\right)$ and $K_{4 \rho}\left(x_{o}\right)$, with the choices

$$
s_{1}=0, \quad t_{1}=c_{1}\left[f_{K_{2 \rho}\left(x_{o}\right)} u_{o} d x\right]^{2-p} \rho^{p}, \quad c_{1}=\frac{2^{(N-1)(2-p)}}{\gamma^{2-p}} .
$$


As a result, if we define $\bar{T}:=t_{1}$, then we have

$$
f_{K_{2 \rho}\left(x_{o}\right)} u_{o} d x \leq 2^{N+1} \gamma \inf _{0<\tau<\bar{T}} f_{K_{4 \rho}\left(x_{o}\right)} u(x, \tau) d x .
$$

It is well-known that solutions to (2.3) extinguish in finite time. The estimate (2.5) shows solutions will not extinguish before $\bar{T}$.

\subsection{A Harnack-type Inequality when $1<p \leq p_{*}$}

We recall here the main result of [7]. The supremum or infimum of a function is meant to be the essential one. Moreover, we work in general, open, bounded sets $\Omega \subset \mathbb{R}^{N}$ and $\Omega_{S}:=\Omega \times[0, S]$. At this step, there is no connection between $\Omega$ and the set $E$ we have considered so far.

Theorem 2.1. Let $u$ be a non-negative, locally bounded, local, weak solution to (1.1)(1.2), (1.4) -(1.5) in $\Omega_{S}$, with $p$ satisfying (1.3), and let $r \geq 1$ be such that

$$
\lambda_{r} \stackrel{\text { def }}{=} N(p-2)+r p
$$

is strictly positive. Let $(y, s) \in \Omega_{S}$, consider $\rho>0$ such that $K_{2 \rho}(y) \subset \Omega$, and set

$$
\theta=c_{2}\left[f_{K_{2 \rho}(y)} u(x, s) d x\right]^{2-p}, \quad \sigma=\left[\frac{f_{K_{2 \rho}(y)} u(x, s) d x}{\left(f_{K_{2 \rho}(y)} u^{r}(x, s) d x\right)^{\frac{1}{r}}}\right]^{\frac{p r}{\lambda_{r}}} .
$$

Then there exist a constant $c_{2} \in(0,1)$ that can be determined only in terms of the data $\left\{p, N, C_{o}, C_{1}\right\}$, and two positive constants $\gamma$ and $d$, that can be determined in terms of the data $\left\{p, N, C_{o}, C_{1}\right\}$ and $r$, such that if $K_{16 \rho}(y) \times\left[s, s+\theta \rho^{p}\right] \subset \Omega_{S}$, then there holds

$$
\inf _{K_{2 \rho}(y) \times\left[s+\frac{3}{4} \theta \rho^{p}, s+\theta \rho^{p}\right]} u \geq \gamma \sigma^{d} \sup _{K_{\rho}(y) \times\left[s+\frac{1}{2} \theta \rho^{p}, s+\theta \rho^{p}\right]} u,
$$

Remark 2.4. We point out that Theorem 2.1 holds not just for $p$ in (1.3), but for any pair of $p \in(1,2)$ and $r \geq 1$, such that $\lambda_{r}>0$. See the discussion following Theorem 1.1 in [7]. Here we limited ourselves to the statement which is needed in our context.

Remark 2.5. The statement given in [7] is slightly different, as far as the top of the cylinder, where the infimum is taken, is concerned. Indeed, Theorem 1.1 of [7] states that

$$
\inf _{K_{2 \rho}(y) \times\left[s+\frac{3}{4} \theta \rho^{p}, s+\left(\frac{3}{4}+\frac{1}{4^{p+1}}\right) \theta \rho^{p}\right]} u \geq \gamma \sigma^{d} \sup _{K_{\rho}(y) \times\left[s+\frac{1}{2} \theta \rho^{p}, s+\theta \rho^{p}\right]} u .
$$

However, a careful inspection of the proof given in [7], and a comparison with the proof of the analogous result given in [6, Chapter 6, Section 11], shows that the top of the cylinders can be taken to be the same, both for the supremum and the infimum. We refrain from giving further details here. 


\subsection{A Lower Bound on the Super-Solution $v$ Defined in (2.1)}

From here on we let

$$
c \stackrel{\text { def }}{=} \min \left\{c_{1}, c_{2}\right\},
$$

which are given in (2.4) and in (2.6), and $\bar{T}$ is accordingly redefined through $c$. We point out that either it remains the same, or it is shortened. The following lemma has been shown in [9, Lemma 5.1].

Lemma 2.3. Let $Q, u_{k}, \mu, v$ be as in $\S$ 2.2. consider $\left(x_{o}, \eta\right) \in \Sigma$ with $s<\eta \leq t_{o}$, let

$$
\theta=c\left[f_{K_{2 \rho}\left(x_{o}\right)} v(x, \eta) d x\right]^{2-p}
$$

and assume that

$$
s \leq \eta-\theta \rho^{p}<\eta \leq t_{o} .
$$

Then, if we let

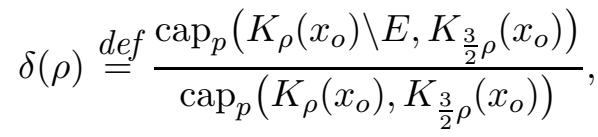

there exists a constant $\gamma>1$ that depends only on the data $\left\{p, N, C_{o}, C_{1}\right\}$, such that

$$
\mu[\delta(\rho)]^{\frac{1}{p-1}} \leq \gamma f_{K_{2 \rho}\left(x_{o}\right)} v(x, \eta) d x .
$$

Remark 2.6. Lemma 2.3 holds for all $1<p<2$, and does not depend on conditions (1.4)-(1.5).

\section{Proof of Theorem 1.1}

Consider (2.3) with $u_{o}(x)=v(x, \eta) \chi_{K_{2 \rho}\left(x_{o}\right)}(x)$. Assume that

$$
s<\eta<\eta+\bar{T} \leq t_{o}, \quad \text { where } \bar{T}=c\left[f_{K_{2 \rho}\left(x_{o}\right)} v(x, \eta) d x\right]^{2-p} \rho^{p} .
$$

According to (2.5), we have

$$
\frac{1}{2^{N+1} \gamma} f_{K_{2 \rho}\left(x_{o}\right)} u_{o}(x) d x \leq \inf _{0<\tau<\bar{T}} f_{K_{4 \rho}\left(x_{o}\right)} u(x, \tau) d x .
$$

This together with (2.7) yields

$$
\sup _{K_{4 \rho}\left(x_{o}\right) \times\left[\frac{1}{2} \bar{T}, \bar{T}\right]} u \geq \frac{1}{2^{N+1} \gamma} f_{K_{2 \rho}\left(x_{o}\right)} u_{o}(x) d x
$$




$$
\begin{aligned}
& =\frac{1}{2^{N+1} \gamma} f_{K_{2 \rho}\left(x_{o}\right)} v(x, \eta) d x \\
& \geq \gamma \mu[\delta(\rho)]^{\frac{1}{p-1}}
\end{aligned}
$$

where $\gamma$ in the last line takes into account all the constants.

We can now apply Theorem 2.1 with $\Omega=K_{32 \rho}\left(x_{o}\right)$ and $(y, s)=\left(x_{o}, 0\right)$, to conclude that

$$
\inf _{K_{2 \rho}\left(x_{o}\right) \times\left[\frac{3}{4} \bar{T}, \bar{T}\right]} u \geq \gamma \sigma^{d} \mu[\delta(\rho)]^{\frac{1}{p-1}}
$$

where

$$
\sigma \stackrel{\text { def }}{=}\left[\frac{f_{K_{2 \rho}\left(x_{o}\right)} u_{o} d x}{\left(f_{K_{2 \rho}\left(x_{o}\right)} u_{o}^{r} d x\right)^{\frac{1}{r}}}\right]^{\frac{p r}{\lambda_{r}}},
$$

$r>1$ is such that $\lambda_{r}>0$, and $d$ depends on the data and on $r$. Since

$$
f_{K_{2 \rho}\left(x_{o}\right)} u_{o} d x \stackrel{\sqrt{2.7})}{\geq} \frac{1}{\gamma} \mu[\delta(\rho)]^{\frac{1}{p-1}}, \quad\left(f_{K_{2 \rho}\left(x_{o}\right)} u_{o}^{r} d x\right)^{\frac{1}{r}} \leq \mu^{\frac{r-1}{r}}\left(f_{K_{2 \rho}\left(x_{o}\right)} u_{o} d x\right)^{\frac{1}{r}},
$$

we eventually obtain

$$
\inf _{K_{2 \rho}\left(x_{o}\right) \times\left[\frac{3}{4} \bar{T}, \bar{T}\right]} u \geq \gamma \mu[\delta(\rho)]^{\frac{1}{p-1}\left(1+d \frac{p(r-1)}{\lambda_{r}}\right)} .
$$

By Lemma 2.1 and Remark 2.1, we have $v(x, \tau+\eta) \geq u(x, \tau)$ a.e. in $K_{32 \rho}\left(x_{o}\right) \times[0, \bar{T}]$. Therefore, setting

$$
q_{o} \stackrel{\text { def }}{=} \frac{1}{p-1}\left(1+d \frac{p(r-1)}{\lambda_{r}}\right)
$$

we conclude

$$
\mu[\delta(\rho)]^{q_{o}} \leq \gamma \inf _{K_{2 \rho}\left(x_{o}\right)} v(\cdot, \tau)
$$

for all

$$
\tau \in\left[\eta+\frac{3}{4} \theta \rho^{p}, \eta+\theta \rho^{p}\right], \quad \text { with } \quad \theta=c\left[f_{K_{2 \rho}\left(x_{o}\right)} v(x, \eta) d x\right]^{2-p} .
$$

Since (3.2)-(3.3) correspond to (5.2)-(5.3) of [9], from here on we can conclude the proof as in [9, Section 6], up to minor adaptations of the main argument, which we now discuss.

A key step of the induction argument in [9, Section 6.3] is to fit a sequence of intrinsically scaled cylinders in one another. There we chose to present this trick in a sequence of centered cylinders, such that the oscillation of $u$ is quantitatively reduced 
therein. Here we take the opportunity to point out that actually we may choose a nested sequence of backward cylinders which will do the same job. The modification is based on the following lemma.

Lemma 3.1. Fix $\left(x_{o}, t_{o}\right) \in S_{T}, s$ such that $0<s<t_{o} \leq T$, let $Q, u_{k}, \mu, v$ be as in $\S$ 2.2. consider $\widetilde{\theta}=c \mu^{2-p}$ with $c$ as in $\S$ 2.6, and assume that

$$
s \leq t_{o}-2 \widetilde{\theta}(2 \rho)^{p}<t_{o} .
$$

Then, there exists a constant $\widetilde{\gamma}>1$ that depends only on the data $\left\{p, N, C_{o}, C_{1}\right\}$, such that

$$
\mu[\delta(\rho)]^{q_{o}} \leq \widetilde{\gamma} \inf _{K_{2 \rho}\left(x_{o}\right)} v(\cdot, \tau) \quad \text { for all } \tau \in\left[t_{o}-2 \widetilde{\theta} \rho^{p}, t_{o}\right]
$$

Proof. For simplicity, let us assume (3.2) holds for $\tau=\eta+\theta \rho^{p}$, where $\theta$ is defined in (3.3). Consider the closed and bounded interval

$$
I \stackrel{\text { def }}{=}\left[t_{o}-3 \widetilde{\theta} \rho^{p}, t_{o}\right] \subset\left[t_{o}-2 \widetilde{\theta}(2 \rho)^{p}, t_{o}\right] .
$$

Using $0 \leq v \leq \mu$, it is not hard to see that, for any $\eta \geq t_{o}-3 \widetilde{\theta} \rho^{p}$, we have

$$
\eta-c\left[f_{K_{2 \rho}\left(x_{o}\right)} v(x, \eta) d x\right]^{2-p} \rho^{p} \geq t_{o}-2 \widetilde{\theta}(2 \rho)^{p} .
$$

Consequently, Lemma 2.3 can be applied for all $\eta \in I$.

Next, we introduce the function $f: I \rightarrow \mathbb{R}$ defined by

$$
f(\eta)=\eta+c\left[f_{K_{2 \rho}\left(x_{o}\right)} v(x, \eta) d x\right]^{2-p} \rho^{p} .
$$

It is straightforward to check that $f \in C(I)$. Using $0 \leq v \leq \mu$ again, a simple calculation yields that

$$
\begin{aligned}
& \min _{I} f \leq f\left(t_{o}-3 \widetilde{\theta} \rho^{p}\right) \leq t_{o}-2 \widetilde{\theta} \rho^{p}, \\
& \max _{I} f \geq f\left(t_{o}\right) \geq t_{o} .
\end{aligned}
$$

Thus, by the mean value theorem, there exists $t_{*} \in I$ such that $f\left(t_{*}\right)=t_{o}$. Without loss of generality, we may assume $t_{*}$ is the smallest among such numbers in $I$. Accordingly, we define $I_{*}:=\left[t_{o}-3 \widetilde{\theta} \rho^{p}, t_{*}\right]$ and $J:=\left[t_{o}-2 \widetilde{\theta} \rho^{p}, t_{o}\right]$. As a result, there holds $J \subset f\left(I_{*}\right)$, and we can conclude that $f(\cdot)$ attains all values of $J$ as its argument ranges over $I_{*}$. Moreover, since $t_{*}$ is the first time when $f\left(t_{*}\right)=t_{o}$ holds, we must have $f(\eta)<t_{o}$ for all $\eta \in I_{*} \backslash\left\{t_{*}\right\}=\left[t_{o}-3 \widetilde{\theta} \rho^{p}, t_{*}\right)$. Consequently, we are allowed to apply (3.2)-(3.3) for all $\eta \in I_{*}$, and conclude that

$$
\mu[\delta(\rho)]^{q_{o}} \leq \widetilde{\gamma} \inf _{K_{2 \rho}} v(\cdot, \tau) \quad \text { for all } \tau \in\left[t_{o}-2 \widetilde{\theta} \rho^{p}, t_{o}\right],
$$

for some properly defined positive constant $\widetilde{\gamma}$. 
Remark 3.1. Due to the estimates we relied upon, it is apparent that we have a sharp disconnect in the characterization of the boundary behavior between $p \in\left(1, p_{*}\right]$ considered here, and $p>p_{*}$ studied in [9, 10].

Remark 3.2. If (1.1) reduces to the prototype parabolic $p$-laplacian (1.13), then by (2.10) of [4], $d$ has an explicit expression, namely $d=1+\frac{\lambda_{r}}{\operatorname{pr(2-p)}}$, and consequently also $q_{o}$ in (3.1).

\section{Proof of Corollary 1.2}

We first present the main ingredient of the proof in the following Lemma 4.1, which plays a similar role in the induction argument as Lemma 3.1. Meanwhile, we emphasize that Lemma 4.1 holds for all $1<p<2$ without conditions (1.4)-(1.5). Instead of Harnack's inequality, we employ the expansion of positivity for non-negative supersolutions to (1.1)-(1.2); see [6, Chapter 4, Proposition 5.1].

Lemma 4.1. Let $Q, u_{k}, \mu, v$ be as in $\S$ 2.2. consider $\left(x_{o}, t_{o}\right) \in \Sigma$ with $s<t_{o}$, let $\widetilde{\theta}=c \mu^{2-p}$ with $c$ as in $\S$ [2.6, assume that

$$
s \leq t_{o}-2 \widetilde{\theta}(2 \rho)^{p}<t_{o},
$$

and that the complement of $E$ is uniformly $p$-fat. Then, there exists a constant $\widetilde{\gamma}>1$ that depends only on the data $\left\{p, N, C_{o}, C_{1}\right\}$ and $\gamma_{o}$, such that for all $0<\rho<\bar{\rho}$,

$$
\mu \leq \widetilde{\gamma} \inf _{K_{2 \rho}\left(x_{o}\right)} v(\cdot, \tau) \quad \text { for all } \tau \in\left[t_{o}-2 \widetilde{\theta} \rho^{p}, t_{o}\right] .
$$

Proof. As in the proof of Lemma 3.1, we define the interval $I$, the function $f \in C(I)$, and verify that Lemma 2.3 can be applied, i.e.

$$
\mu[\delta(\rho)]^{\frac{1}{p-1}} \leq \gamma f_{K_{2 \rho}\left(x_{o}\right)} v(x, \tau) d x \quad \text { for all } \tau \in I=\left[t_{o}-3 \widetilde{\theta} \rho^{p}, t_{o}\right] .
$$

Our proof departs from here. Let $\sigma \in(0,1)$ to be fixed later. We use the uniform $p$-fatness of the complement of $E$ to estimate for all $\tau \in I$ and $0<\rho<\bar{\rho}$,

$$
\begin{aligned}
\mu \gamma_{o}^{\frac{1}{p-1}} & \leq \gamma f_{K_{2 \rho}\left(x_{o}\right)} v(x, \tau) d x \\
& =\frac{\gamma}{\left|K_{2 \rho}\right|} \int_{K_{2 \rho}\left(x_{o}\right)} v(x, \tau) \chi_{[v>\sigma \mu]} d x+\frac{\gamma}{\left|K_{2 \rho}\right|} \int_{K_{2 \rho}\left(x_{o}\right)} v(x, \tau) \chi_{[v \leq \sigma \mu]} d x \\
& \leq \gamma \mu \frac{\left|[v(\cdot, \tau)>\sigma \mu] \cap K_{2 \rho}\left(x_{o}\right)\right|}{\left|K_{2 \rho}\right|}+\gamma \sigma \mu .
\end{aligned}
$$

Now we choose $\gamma \sigma=\frac{1}{2} \gamma_{o}^{\frac{1}{p-1}}$, and after simple manipulation we arrive at

$$
\left|[v(\cdot, \tau)>\sigma \mu] \cap K_{2 \rho}\left(x_{o}\right)\right| \geq \sigma\left|K_{2 \rho}\right| \quad \text { for all } \tau \in I .
$$

Since $v$ is a non-negative super-solution to (1.1)-(1.2) in $Q$, an application of [6, Chapter 4, Proposition 5.1] yields the desired conclusion. 
Based on Lemma 4.1, the proof of Corollary 1.2 can be completed by an induction argument, just like in [9]: we refrain from further elaboration, to avoid repetition.

\section{Appendices}

\section{A Capacity and Potential Theory}

The concept of capacity plays a key role in potential theory and particularly, in understanding the local behavior of Sobolev functions. In a sense, it takes the place of measure in Egorov and Lusin type theorems for Sobolev functions. Decisive here for the boundary behavior of solutions to the parabolic $p$-laplacian type equations is the $p$-capacity defined by

$$
\operatorname{cap}_{p}(K, \Omega)=\inf _{u \in W} \int_{\Omega}|D u|^{p} d x
$$

where $K$ is a compact subset of the open set $\Omega$ in $\mathbb{R}^{N}$ and $W=\left\{u \in C_{o}^{\infty}(\Omega)\right.$ : $u \geq 1$ on $K\}$. After proper approximations, the above minimization could take place over $W_{o}^{1, p}(\Omega)$ instead of $C_{o}^{\infty}(\Omega)$ and the capacity of an arbitrary subset of $\Omega$ can be formulated based on that of compact subsets, cf. [11, Chapter 2] and [15, Chapter 2].

For a domain $E \subset \mathbb{R}^{N}$ and a point $x \in \mathbb{R}^{N}$, in potential theory (cf. 11, Definition 6.3.7] and [15, Definition 2.47]) we say $E$ is $p$-thick at $x$ if

$$
\int_{0}^{1}\left[\frac{\operatorname{cap}_{p}\left(K_{\rho}(x) \cap E, K_{\frac{3}{2} \rho}(x)\right)}{\operatorname{cap}_{p}\left(K_{\rho}(x), K_{\frac{3}{2} \rho}(x)\right)}\right]^{\frac{1}{p-1}} \frac{d \rho}{\rho}=\infty .
$$

Even though the introduction of this concept was motivated by Wiener's celebrated work [17] about the boundary regularity for the laplacian, nevertheless it has witnessed a development largely independent of the theory of partial differential equations. It is vacuous that the domain $E$ is $p$-thick at its interior point, while not so for points in $\bar{E}^{c}$. Hence the most interesting part concerning thickness lies in the boundary $\partial E$. A deep result in potential theory-the Kellogg property-asserts that the domain $E$ is $p$-thick at every point of $\partial E$ except a set of zero $p$-capacity, cf. [1, $\S 6.3]$.

The peculiar feature of the notion of thickness is that it is purely geometric; unfortunately, apart from easy situations, it is far from trivial to provide explicit examples of $p$-thickness. Perhaps, one of the most interesting cases is given by the so-called outer corkscrew condition (OCC): we say that $x_{o} \in \partial E$ satisfies (OCC), if there exist $M \geq 2$ and $r_{o}>0$, such that for any $r \in\left(0, r_{o}\right)$ there exists $a_{r} \in E^{c}$ satisfying $\frac{r}{M}<\left|a_{r}-x_{o}\right|<r$ and $\operatorname{dist}\left(a_{r}, \partial E\right)>\frac{r}{M}$. Then, by [11, Theorem 6.31] it is not hard to prove that $x_{o}$ is a Wiener point. A domain $E$ that has an outward spike satisfies an outer corkscrew condition at the tip of the spike. Other examples of Wiener points are discussed in [2, Corollary 11.25]. 


\section{References}

[1] D.R. Adams and L.I. Hedberg, "Function spaces and potential theory", Grundlehren der Mathematischen Wissenschaften, 314, Springer-Verlag, Berlin, 1996.

[2] A. Björn and J. Björn, "Nonlinear potential theory on metric spaces", EMS Tracts in Mathematics 17, European Mathematical Society, Zürich, 2011.

[3] A. Björn, J. Björn, U. Gianazza and M. Parviainen, Boundary regularity for degenerate and singular parabolic equations, Calc. Var. Partial Differential Equations, 52(3), (2015), 797-82\%.

[4] M. Bonforte, R.G. Iagar and J.L. Vázquez, Local smoothing effects, positivity, and Harnack inequalities for the fast $p$-Laplacian equation, Adv. Math., 224(5), (2010), 2151-2215.

[5] E. DiBenedetto, "Degenerate Parabolic Equations", Universitext, Springer-Verlag, New York, 1993.

[6] E. DiBenedetto, U. Gianazza and V. Vespri, "Harnack's Inequality for Degenerate and Singular Parabolic Equations", Springer Monographs in Mathematics, Springer-Verlag, New York, 2012.

[7] S. Fornaro and V. Vespri, Harnack estimates for non-negative weak solutions of a class of singular parabolic equations, Manuscripta Math., 141(1-2), (2013), 85103.

[8] R.F. Gariepy and W.P. Ziemer, A regularity condition at the boundary for solutions of quasilinear elliptic equations, Arch. Rational Mech. Anal., 67(1), (1977), 25-39.

[9] U. Gianazza, N. Liao and T. Lukkari, A boundary estimate for singular parabolic diffusion equations, NoDEA Nonlinear Differ. Equ. Appl., 4, Art. 33, (2018), $24 p p$.

[10] U. Gianazza and N. Liao, A boundary estimate for degenerate parabolic diffusion equation, Potential Anal., 53(3), (2020), 977-995.

[11] J. Heinonen, T. Kilpeläinen and O. Martio, "Nonlinear Potential Theory of Degenerate Elliptic Equations", 2nd ed., Dover, Mineola, NY, 2006.

[12] T. Kilpeläinen and P. Lindqvist, On the Dirichlet boundary value problem for a degenerate parabolic equation, SIAM J. Math. Anal., 27, (1996), 661-683.

[13] R. Korte, T. Kuusi and M. Parviainen, A connection between a general class of superparabolic functions and supersolutions, J. Evol. Equ., 10, (2010), 1-20. 
[14] J. L. Lewis, Uniformly fat sets, Trans. Amer. Math. Soc., 308, (1988), 177-196.

[15] J. Malý and W.P. Ziemer, "Fine regularity of solutions of elliptic partial differential equations", Mathematical Surveys and Monographs, 51, American Mathematical Society, Providence, RI, 1997.

[16] V.G. Maz'ya, The continuity at a boundary point of the solutions of quasi-linear elliptic equations, Vestnik Leningrad Univ. Math., 25(13), (1970), 42-55.

[17] N. Wiener, The Dirichlet Problem, J. Math. and Phys., 3(3), (1924), 127-146. 